\title{
On the scaling of shear-driven entrainment: a DNS study
}

\author{
HARM J. J. JONKER ${ }^{1} \dagger$ MAARTEN VAN REEUWIJK ${ }^{2}$, \\ PETER P. SULLIVAN ${ }^{3}$, AND EDWARD G. PATTON \\ ${ }^{1}$ Delft University, Netherlands \\ ${ }^{2}$ Department of Civil and Environmental Engineering, Imperial College, UK \\ ${ }^{3}$ National Center for Atmospheric Research, Colorado, USA
}

(Received ?; revised ?; accepted ?. - To be entered by editorial office)

The deepening of a shear-driven turbulent layer penetrating into a stably stratified quiescent layer is studied using Direct Numerical Simulation (DNS). The simulation design mimics the classical laboratory experiments by Kato and Phillips (J. Fluid Mech. 37, 643-655, 1969) in that it starts with linear stratification and applies a constant shear stress at the lower boundary, but avoids side wall and rotation effects inherent in the original experiment. It is found that the layers universally deepen as a function of the square root of time, independent of the initial stratification and the Reynolds number of the simulations, provided that the Reynolds number is large enough. Consistent with this finding, the dimensionless entrainment velocity varies with the bulk Richardson number as $R i^{-\frac{1}{2}}$. In addition it is observed that all cases evolve in a self-similar fashion. A selfsimilarity analysis of the conservation equations shows that only a square root growth law is consistent with self-similar behaviour.

Key words: Authors should not enter keywords on the manuscript; must be chosen during online submission.

\section{Introduction}

An important issue in geophysical flows is the deepening rate of a turbulent fluid layer growing into a quiescent stably stratified layer through the process of turbulent entrainment (Fernando 1991). The turbulence can be generated by different mechanisms, e.g. buoyancy or shear. Here we focus on the shear-driven case. Relevant examples are deepening oceanic boundary layers where surface winds create a shear stress at the water surface (Thorpe 2005; Price 1981), or wind-driven atmospheric boundary layers where shear is created due to surface friction (Stull 1998). The measurement of turbulent entrainment in a geophysical context is challenging because entrainment fluxes are generally small quantities easily overwhelmed by large-scale vertical motions and because boundary and initial conditions cannot be controlled or are not fully known. Consequently, there has been a concerted effort to study turbulent entraining boundary layers in well controlled laboratory experiments. An ingenious laboratory experiment of this type was conceived by Kato \& Phillips (1969), hereafter referred to as KP69. The experimental apparatus had the shape of an annulus so as to simulate turbulent entrainment in an unbounded setting. A fixed shear stress, characterised by the friction velocity $u_{*}$, was imposed by

$\dagger$ Email address for correspondence: h.j.j.jonker@tudelft.nl 


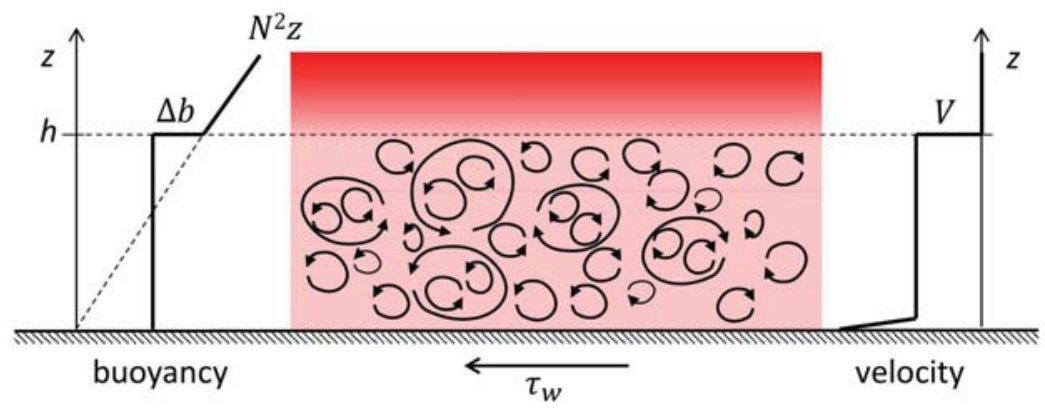

FiguRE 1. Sketch of the deepening of a shear driven mixed layer into linearly stratified fluid layer.

lowering a screen on to the water surface and spinning the screen with constant torque. The initial density distribution $\rho(z)$, characterized by the buoyancy $b=-g\left(\rho-\rho_{0}\right) / \rho_{0}$ where $\rho_{0}$ is a reference density, was linear $b=N^{2} z$, where $N$ is the buoyancy frequency $N=(\partial b / \partial z)^{1 / 2}$.

Due to the shearing a turbulent mixed layer of depth $h(t)$ forms which grows in time at an entrainment rate $w_{e} \equiv \mathrm{d} h / \mathrm{d} t$. Throughout this study we will associate $h$ with the location of the maximum gradient in the buoyancy profile $b(z)$, see also $\S 3.2$. Under the assumption that viscous transport effects are negligible, i.e. that the Reynolds number is large enough, it is expected that

$$
\frac{w_{e}}{u_{*}}=E(R i)
$$

where $E$ is the normalized entrainment rate and $R i$ is the Richardson number which measures the stability of the density interface with respect to $u_{*}$ and is defined as

$$
R i=h \Delta b / u_{*}^{2},
$$

where $\Delta b$ is the buoyancy jump at the top of the mixed layer. Within the context of a well mixed layer with depth $h$, for a linear initial stratification the buoyancy difference between the mixed and quiescent layer is $\Delta b=N^{2} h / 2$, i.e. $R i=N^{2} h^{2} /\left(2 u_{*}^{2}\right)$; see figure 1. Note that $R i$ is not constant in this set-up because $h=h(t)$. Although there was some significant scatter in the data, according to KP69 the power law most representative of the data spanning the range $10<R i<400$ was $E=2.5 R i^{-1}$. The -1 exponent appeared consistent with an energy conservation argument matching the turbulence production to the work required for raising the potential energy of the system (see also §5). The KP69 data has been used to validate several turbulence models for geophysical flows (e.g. Mellor \& Durbin 1975; Kundu 1981; Galperin et al. 1988).

A second type of entrainment experiment carried out in this type of apparatus used a two-layer stratification as the initial condition (Kantha et al. 1977). These experiments have the advantage that $R i$ remains constant over the duration of the experiment, thereby allowing more accurate measurement of $w_{e}$. Kantha et al. (1977) found entrainment rates that were significantly higher than those in KP69, which they attributed to the absence of internal waves in the two-layer set-up. Furthermore, they concluded that there was no single power-law fit for $E$ in the measurement range $30<R i<1000$.

The two-layer set-up became the preferred entrainment experiment in subsequent years, during which it became evident that several other factors influence $E$ in the annulus set-up; for example, the finite annulus width $W$ introduces friction along the side walls, meaning that not all of the imparted energy from the screen is available for 
turbulent entrainment. Price (1979) developed a bulk model incorporating the side wall influence and concluded that the flow dynamics inside the system also depend on $h / W$ and a Richardson number that accounts for the mean velocity jump $V$ across the density interface (see figure 1)

$$
R i_{v}=h \Delta b / V^{2}
$$

thus suggesting that

$$
\frac{w_{e}}{u_{*}}=E\left(R i, R i_{v}, \frac{h}{W}\right) .
$$

In the limit of $h / W \rightarrow 0$, Price (1979) showed that the expected power-law is $E=$ $n R i_{v}^{1 / 2} R i^{-1 / 2}$ where $n=1 / 2$ or 1 for linear and two-layer stratification, respectively. Price (1979) observed that $R i_{v} \approx 0.6$ over a wide range of $R i$, which leads to $E=$ $0.38 R i^{-1 / 2}$ for the KP69 set-up; careful comparison showed that this entrainment law was not inconsistent with the KP69 data during the initial phase of the experiment when side wall effects are still negligible. This entrainment law is very similar to an earlier mixed layer model developed by Pollard et al. (1973), which led to the prediction $E=\frac{1}{2} R i^{-1 / 2}$. Thompson (1979) re-analyzed the KP69 and Kantha et al. (1977) data and independently concluded that the data supported the theory of Pollard et al. (1973) in the early phases of the experiments, i.e. before sidewall friction became important. Later experiments by Deardorff \& Willis (1982) also confirmed this, although they obtained an entrainment law $E \propto R i_{v}^{-1.4} R i^{-1 / 2}$.

Furthermore, it was discovered that the annular geometry creates secondary currents (Scranton \& Lindberg 1983) and that the interface could tilt significantly under certain circumstances. Moreover, Scranton \& Lindberg (1983) observed that the turbulence levels in the outer $15 \%$ of the annulus were of a much higher intensity than for the inner $85 \%$. This was studied in more detail by Deardorff \& Yoon (1984), who attributed this effect to turbulence damping within the mixed layer because of inertial stability associated with angular momentum increasing rapidly with radius except very close to the outer wall. Size effects were also significant: Deardorff \& Yoon (1984) report that Kantha in 1978 performed experiments with an annulus half the size of KP69, and saw a significant reduction in the turbulent entrainment. Jones \& Mulhearn (1983) performed experiments with an annulus about twice the size of the one used by KP69 and concluded by comparing their data together with that of KP69 and Kantha et al. (1977) that a Richardson number based on the annulus width $W$ gave the best collapse of the entrainment data.

Clearly, much progress has been made in understanding turbulent entrainment in annuli, but owing to geometrical and finite size effects the relation to the atmospheric and oceanic counterparts has remained much weaker than hoped for. Indeed, the original question "what is the entrainment law for a linearly stratified fluid subjected to shearing at one boundary?" remains open after more than forty years. In this paper, we aim to resolve this question by using Direct Numerical Simulation. Although the set-up of the runs are largely inspired by the KP69 set-up, we target an even purer case with DNS, to the extent that our set-up includes no rotational effects and no side walls which spuriously influence the entrainment results. Such a case was termed "the ideal experiment" by Price (1979). We will limit ourselves here to unit Prandtl number. 


\section{Simulation design}

We solve the incompressible Navier-Stokes equations in the Boussinesq approximation, given by

$$
\begin{aligned}
\frac{\partial \mathbf{u}}{\partial t}+\mathbf{u} \cdot \nabla \mathbf{u} & =-\rho_{0}^{-1} \nabla p+\nu \nabla^{2} \mathbf{u}+b \mathbf{e}_{3} \\
\frac{\partial b}{\partial t}+\mathbf{u} \cdot \nabla b & =\kappa \nabla^{2} b \\
\boldsymbol{\nabla} \cdot \mathbf{u} & =0 .
\end{aligned}
$$

Here, $\mathbf{x}=(x, y, z)$ with $x, y$ the lateral and $z$ the vertical coordinates, $\mathbf{u}=(u, v, w)$ is the fluid velocity, $p$ the pressure, $\mathbf{e}_{3}=(0,0,1), \nu$ and $\kappa$ are the kinematic viscosity and diffusivity, respectively. It is convenient to work with buoyancy because it naturally encompasses situations where density variations are caused by temperature (atmosphere), by salinity concentrations (such as the experiments of KP69) or by a combination of both (ocean). In atmospheric situations one would have $b=g\left(\theta_{\mathrm{v}}-\theta_{\mathrm{v} 0}\right) / \theta_{\mathrm{v} 0}$ with $\theta_{\mathrm{v}}$ the virtual potential temperature and $g$ the gravitational acceleration, whereas $b=-g\left(\rho-\rho_{0}\right) / \rho_{0}$ for salinity; the subscript 0 is used to indicate a reference value. In both situations the linear stratification can be conveniently expressed by the buoyancy frequency $N=(\partial b / \partial z)^{1 / 2}$. The DNS code solves Eqs (2.1-2.3) in a cuboidal domain and is fully parallelized making use of domain decomposition in two directions. The spatial differential operators are discretized using symmetry-preserving second order central differences (Verstappen \& Veldman 2003), and time-integration is carried out with an adaptive second order AdamsBashforth method. Periodic boundary conditions are applied in the lateral directions. Incompressibility (2.3) is enforced by taking the divergence of (2.1) and solving the resulting Poisson equation for $p$. This is done by performing FFTs in the lateral (periodic) directions and subsequently solving a tridiagional system in the vertical direction. Further details can be found in van Reeuwijk (2007); van Reeuwijk et al. (2008).

The initial conditions are given by $\mathbf{u}=0$, and $b(x, y, z)=N^{2} z$. Following the ideas of KP69, we prescribe at the wall a zero buoyancy flux and a constant shear stress, $\tau_{w}=-\rho_{0} u_{*}^{2}$, where $u_{*}$ denotes the (prescribed) friction velocity; this translates to the following Neumann boundary conditions at $z=0: \partial u / \partial z=u_{*}^{2} / \nu, \partial b / \partial z=0$. At the top wall $(z=H)$ a no-slip condition is applied $\mathbf{u}=0$ as well as a very small buoyancy flux to maintain the linear stratification at the top, which would otherwise slowly diffuse due to molecular effects; i.e. we prescribe $\partial b / \partial z=N^{2}$. A random (white noise) perturbation with an amplitude on the order of $N u_{*}$ was added to the initial buoyancy field to aid the transition to turbulence at the onset of the simulation.

Typical scales in this problem are the friction velocity $u_{*}$ and the buoyancy frequency $N$; from these external quantities, one can construct the following time-, length- and buoyancy scales

$$
t_{*}=N^{-1}, \quad h_{*}=u_{*} / N, \quad b_{*}=N u_{*},
$$

and buoyancy Reynolds number $R e_{b}=u_{*} h_{*} / \nu$, i.e.

$$
R e_{b}=\frac{u_{*}^{2}}{N \nu}
$$




\begin{tabular}{|c|c|c|c|c|c|c|c|}
\hline \multicolumn{3}{|c|}{$|\operatorname{run}| u_{*}[\mathrm{~cm} / \mathrm{s}]|H[\mathrm{~cm}]|$} & $N_{x} \times N_{y} \times N_{z}$ & \multicolumn{2}{|c|}{$N^{2}\left[\mathrm{~s}^{-2}\right] \mid h_{*}[\mathrm{~cm}]$} & $T / t_{*} \mid$ & $R e_{b}$ \\
\hline $1 \mathrm{a}$ & 1.00 & 10.4 & $1024 \times 1024 \times 512$ & 7.5 & 0.4 & 210 & 36 \\
\hline $1 b$ & 1.00 & 10.4 & $1024 \times 1024 \times 512$ & 3.75 & 0.5 & 148 & 50 \\
\hline $1 \mathrm{c}$ & 1.00 & 10.4 & $1024 \times 1024 \times 512$ & 1.5 & 0.8 & 94 & 80 \\
\hline $2 \mathrm{a}$ & 3.86 & 64.8 & $2048 \times 2048 \times 1536$ & 7.5 & 1.4 & 210 & 543 \\
\hline $2 \mathrm{~b}$ & 3.86 & 64.8 & $2048 \times 2048 \times 1536$ & 3.75 & 2.0 & 148 & 768 \\
\hline $2 \mathrm{c}$ & 3.86 & 64.8 & $2048 \times 2048 \times 1536$ & 1.5 & 3.1 & 67 & 1214 \\
\hline
\end{tabular}

TABle 1. DNS cases; the domain size is $4 H \times 4 H \times H, \operatorname{Pr}=1$ and $\nu=1 \cdot 10^{-6} \mathrm{~m}^{2} \mathrm{~s}^{-1}$.

It is instructive to briefly consider the dimensionless system

$$
\begin{aligned}
\frac{\partial \tilde{\mathbf{u}}}{\partial \tilde{t}}+\tilde{\mathbf{u}} \cdot \nabla \tilde{\mathbf{u}} & =-\nabla \tilde{p}+R e_{b}^{-1} \nabla^{2} \tilde{\mathbf{u}}+\tilde{b} \mathbf{e}_{3}, \\
\frac{\partial \tilde{b}}{\partial \tilde{t}}+\tilde{\mathbf{u}} \cdot \nabla \tilde{b} & =\left(\operatorname{Pr} R e_{b}\right)^{-1} \nabla^{2} \tilde{b}, \\
\nabla \cdot \tilde{\mathbf{u}} & =0,
\end{aligned}
$$

supplemented with initial condition $\tilde{b}=\tilde{z}$ and boundary conditions $\partial \tilde{u} / \partial \tilde{z}=R e_{b}$, $\partial \tilde{b} / \partial \tilde{z}=0$, at the surface and $\tilde{\mathbf{u}}=0, \partial \tilde{b} / \partial \tilde{z}=1$ at the top. The relevant point here is that besides the Prandtl number (which is taken 1 in this study), the buoyancy Reynolds number $R e_{b}$ is the key parameter characterizing a simulation. Note that $R e_{b}$ remains constant during a simulation whereas $R e_{\tau}=u_{*} h / \nu$ grows significantly. Other dimensionless numbers are the dimensionless domain height $H / h_{*}$ and simulation time $T / t_{*}$, the values of which are not that important as long as they are both large, i.e. a substantial number of time-scales should be simulated while the top of the domain should be high enough not to influence the dynamics.

The DNS runs performed in this study are presented in table 1. Cases 1a,b and c essentially replicate the KP69 experiments (for which $R e_{b}$ was in the range 36 to 201). For cases $2 \mathrm{a}$, b and c, $R e_{b}$ is about a factor 5 larger than the original KP69 experiments. An early DNS study was performed by Ligniéres et al. (1998). Owing to the computational facilities at the time, the simulations were restricted to two dimensions. For the same reason the boundary condition $\partial u / \partial z=u_{*}^{2} / \nu$ was replaced by a pressure gradient force confined to a layer near the surface. This however introduces an extra length scale in the flow set-up. Indeed, the numbers in table 1 show that the present $3 \mathrm{D}$ simulations are computationally intensive even at current standards; also, apart from the grid requirements, the simulations require a very small time step due to the relatively large mean horizontal flow that arises.

\section{Growth rate, entrainment law and rescaled profiles}

\subsection{The relation between $h(t)$ and $E$}

Before discussing the results, we briefly discuss the relation between $h(t)$ and $E$, as these are really two sides of the same coin. As mentioned, the evolution of $h$ depends on $R i$ 


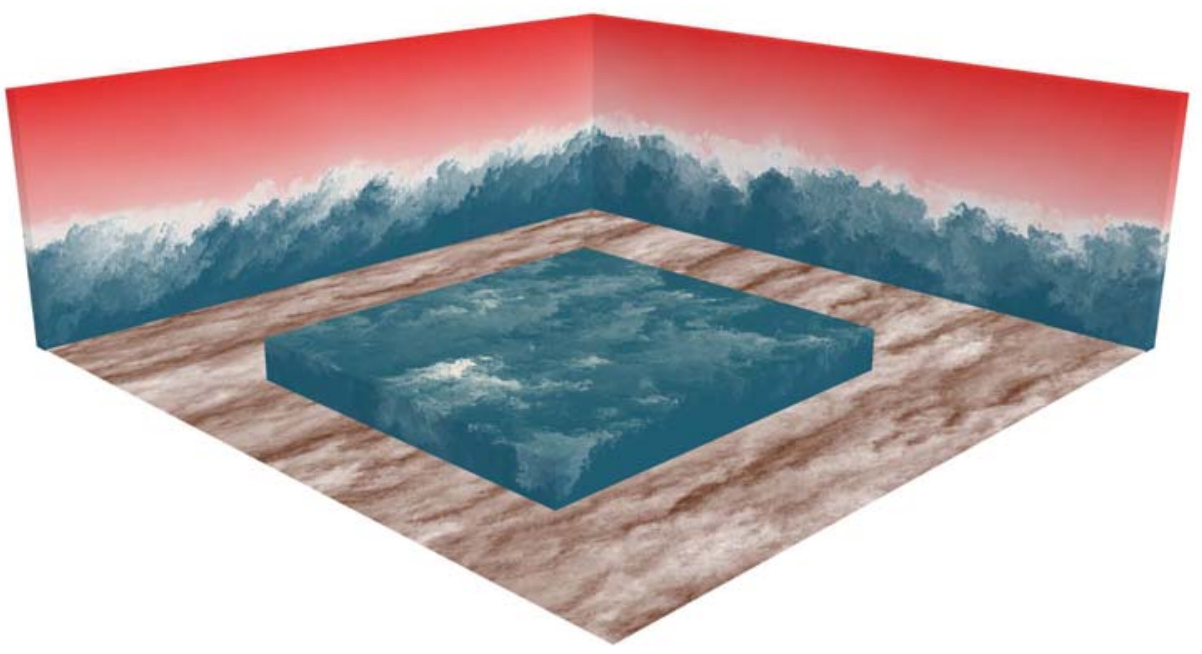

Figure 2. Vertical and horizontal slices of the buoyancy field at the end of simulation 2c. The bottom plane depicts the streaky patterns in the horizontal velocity $u$ just slightly above the surface.

via the entrainment rate $E$. Note that

$$
R i=\frac{N^{2} h^{2}}{2 u_{*}^{2}}=\frac{1}{2}\left(\frac{h}{h_{*}}\right)^{2} .
$$

If one assumes a power law dependence of $E$ on $R i$ with an exponent $a$ - which has yet to be determined- then

$$
E=\frac{w_{e}}{u_{*}}=\frac{1}{u_{*}} \frac{\mathrm{d} h}{\mathrm{~d} t}=c R i^{-a},
$$

and together with (3.1) one can immediately derive that

$$
\frac{h(t)}{h_{*}}=\gamma(N t)^{\alpha}, \quad \alpha=\frac{1}{2 a+1}, \quad \gamma=\left(\frac{2^{a} c}{\alpha}\right)^{\alpha} .
$$

This is interesting because it reveals a direct relation between the entrainment-law (3.2) and the time evolution exponent of $h$; also a relation between the prefactors $c$ and $\gamma$ is established. So if one has determined $\gamma$ and $\alpha$ one also knows $c$ and $a$, or vice versa.

From their experimental results Kato \& Phillips (1969) concluded that $h \sim t^{\frac{1}{3}}$ and $E \sim R i^{-1}$, i.e. $\alpha=1 / 3$ and $a=1$, which is indeed consistent with (3.3). But it should be noted that the predictions by Pollard et al. (1973) leads to $h \sim t^{\frac{1}{2}}$ and $E \sim R i^{-\frac{1}{2}}$, i.e. $\alpha=1 / 2$ and $a=1 / 2$, which are equally consistent with (3.3). The DNS results presented below will shed more light on this issue and serve as a validation of the above models.

\subsection{Simulation results}

Figure 2 shows a snapshot of vertical and horizontal slices of buoyancy at the end of one of the simulations, as well as a slice of the horizontal velocity fluctuations near the surface. To give a general idea of the typical profiles one encounters during the simulations, we have plotted in figure 3 the evolution of the buoyancy and velocity profile of run $1 \mathrm{~b}$; the other runs display profiles with similar shape. For each simulation reported in table 

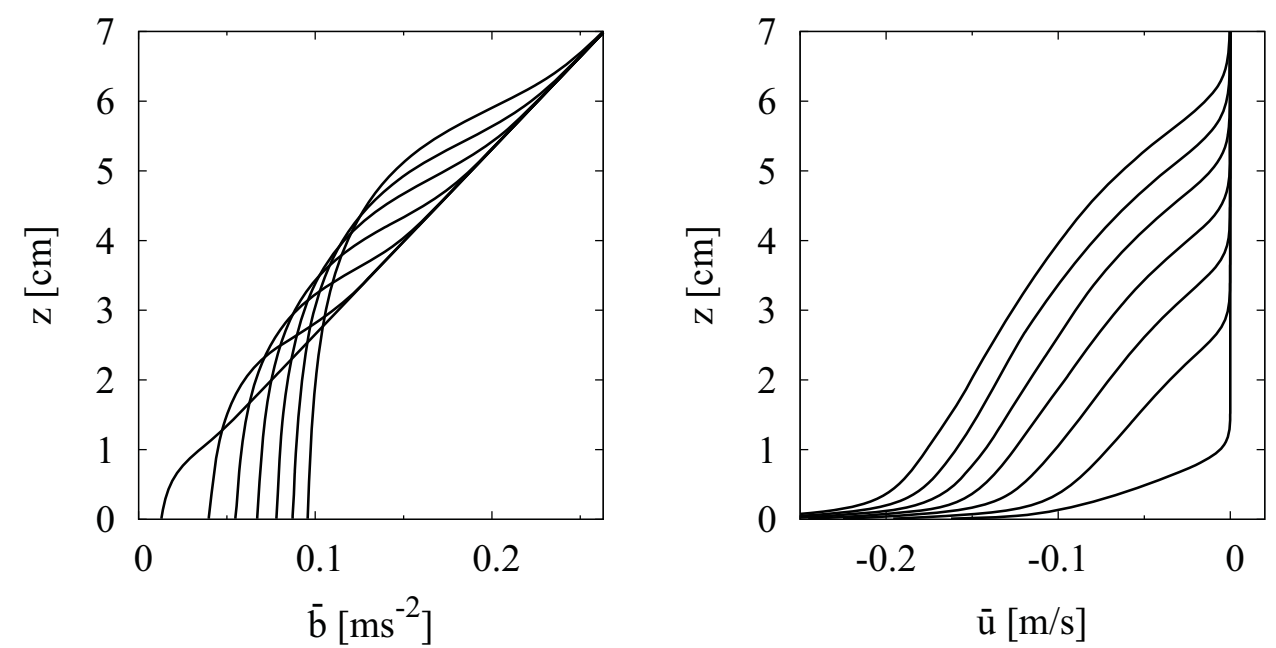

FiguRE 3. Results of simulation $1 \mathrm{~b}$ (see table 1): evolution of the instantaneous horizontally averaged profiles of buoyancy (left) and horizontal velocity (right).

1 , defined by different values of the external parameters $N$ and $u_{*}$, we determined the mixed layer depth $h(t)$ using the method of Sullivan et al. (1998) which entails locating the vertical position where the gradient of the horizontally averaged buoyancy $\partial \bar{b} / \partial z$ is maximum. The left panel of figure 4 shows the diagnosed depth $h$ as a function of time. Note that in these plots no normalization has been applied and one observes in the evolution of $h(t)$ a clear dependence on both the stratification parameter $N$ and the friction velocity $u_{*}$. However, as shown in the right panel of figure 4 , by employing the scaling of (2.4) to $h(t)$ and $t$, all simulations collapse onto a single curve. In particular it is remarkable that no dependence on $R e_{b}$ can be detected in this graph. The second important aspect of this figure is that $\left(h / h_{*}\right)^{2}$ is found to be an almost perfectly linear function of $t$, implying a growth law $h \sim \sqrt{t}$. Setting $\alpha=1 / 2$ in (3.3), this result unambiguously points to an entrainment law of $a=1 / 2$ in (3.2), i.e. $E \sim R i^{-\frac{1}{2}}$.

Note that the data in figure 4 have not been plotted on logarithmic axes, meaning that with very high confidence one can conclude $\alpha=1 / 2$. Fitting the data to a straight line, i.e. presuming $\alpha=1 / 2$, gives a value for the slope $\left(\gamma^{2}\right)$ which is very close to 1 . Using (3.3) with $\gamma \simeq 1$ gives $c=(2 \sqrt{2})^{-1} \approx 0.35$; hence we conclude that the DNS entrainment results are well described by

$$
E=0.35 \frac{1}{\sqrt{R i}} \quad \Leftrightarrow \quad h(t)=\gamma \frac{u_{*}}{N} \sqrt{N t},
$$

with $\gamma \simeq 1.0$.

Upon inspection of figure 5 , one notices that it is also possible to rescale the profiles such that they collapse onto a self-similar profile. Figure 5a for example shows the rescaled buoyancy profile $\bar{b} /\left(N^{2} h\right)$. Because the duration of the simulations measured in dimensionless units $N t$ differs between simulations, our averaging procedure was as follows. First we let transient phenomena subside during $N t<40$ for all simulations. From that instant the profiles were rescaled by the current observed depth $h(t)$ and averaging was carried out until the end of each simulation in two parts, thus yielding two averaged rescaled profiles per simulation. All six simulations have been plotted in this figure and appear to collapse to the same universal profile without any noteworthy dependence on $N, u_{*}$, or time (that is, begin or end part of the averaging period). The same behaviour 

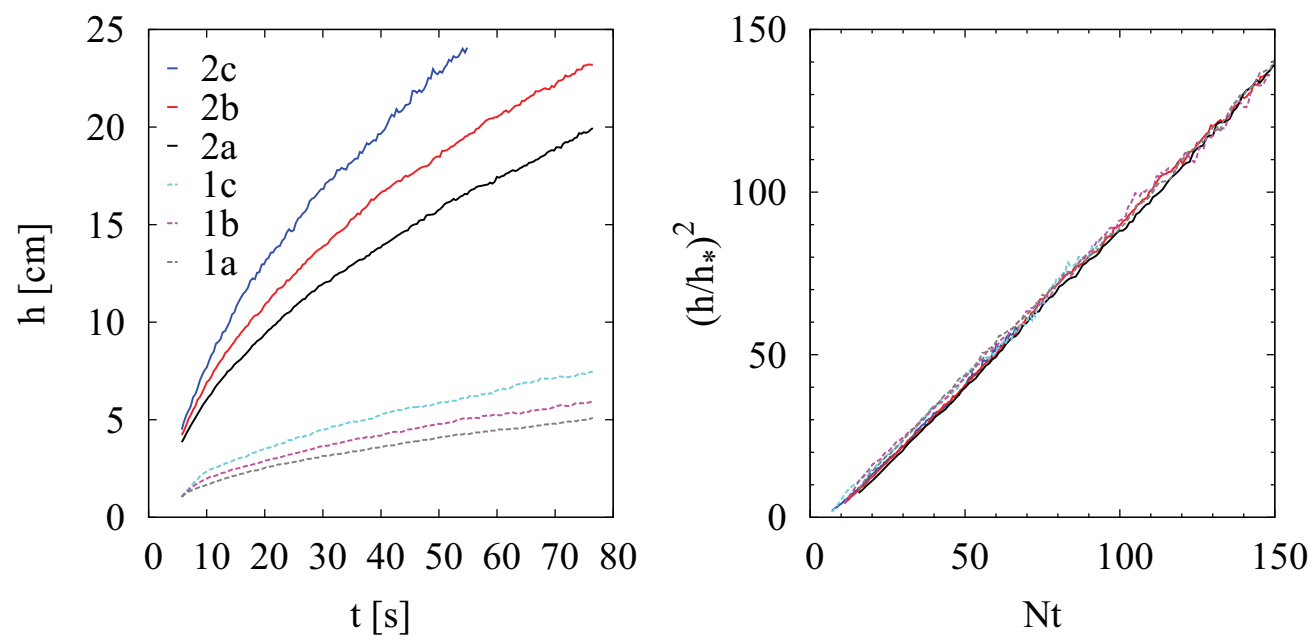

FiguRE 4. Growth of a shear driven boundary layer into a stratified environment. Left: mixed layer depth evolution for the six simulations with varying values of the stratification parameter $N$ and friction velocity $u_{*}$; see table 1 . Right: collapse of the mixed layer depth data after rescaling.

holds for the rescaled velocity profiles $\bar{u} /(N h)$, plotted in figure $5 \mathrm{~b}$, although a somewhat larger deviation can be observed in the lower region of the layer. This deviation occurs because different parameters $\left(\nu\right.$ and $u_{*}$, see $\left.\S 4\right)$ are dominant in the viscous wall region. As time proceeds however, the mixed layer depth continues to increase and gradually the profiles converge to a single universal profile. Figs. 5c,d show that the profiles of the higher order moments, viz. the turbulent buoyancy flux and turbulent momentum flux also exhibit self-similar behaviour.

In figure 6 we have plotted rescaled profiles of turbulence kinetic energy (TKE) $\bar{e}=$ $\left(\overline{u^{\prime 2}}+\overline{v^{\prime 2}}+\overline{w^{\prime 2}}\right) / 2$, the gradient Richardson number $R i_{g}=\partial \bar{b} / \partial z /(\partial \bar{u} / \partial z)^{2}$, the eddy viscosity $K_{m}=-\overline{u^{\prime} w^{\prime}} /(\partial \bar{u} / \partial z)$, and the turbulent Prandtl number $\operatorname{Pr}_{t}$

$$
P r_{t}=\frac{K_{m}}{K_{b}}=\frac{R i_{g}}{R i_{f}}
$$

where $K_{b}=-\overline{w^{\prime} b^{\prime}} /(\partial \bar{b} / \partial z)$ is the eddy diffusivity for buoyancy and $R i_{f}=\overline{w^{\prime} b^{\prime}} /\left(\overline{w^{\prime} u^{\prime}} \partial \bar{u} / \partial z\right)$ is the flux Richardson number. Note that the scaling of the profiles in figure 6 does not involve any time dependence, implying that these quantities are stationary. One observes an effect of the Reynolds number in $\bar{e}$ and $P r_{t}$. It becomes particularly clear from these profiles that the scatter between the low $R e_{b}$ simulations is much larger than the high $R e_{b}$ simulations, which is indicative of the approach to the asymptotic state. Finally it is interesting to note that the shape of the (universal) eddy viscosity profile in figure $6 \mathrm{c}$ is quite similar to the generic profile used in the KPP method (' $K$ profile parameterization') (Large et al. 1994), based on a generic $K$-profile (Troen \& Mahrt 1986). 

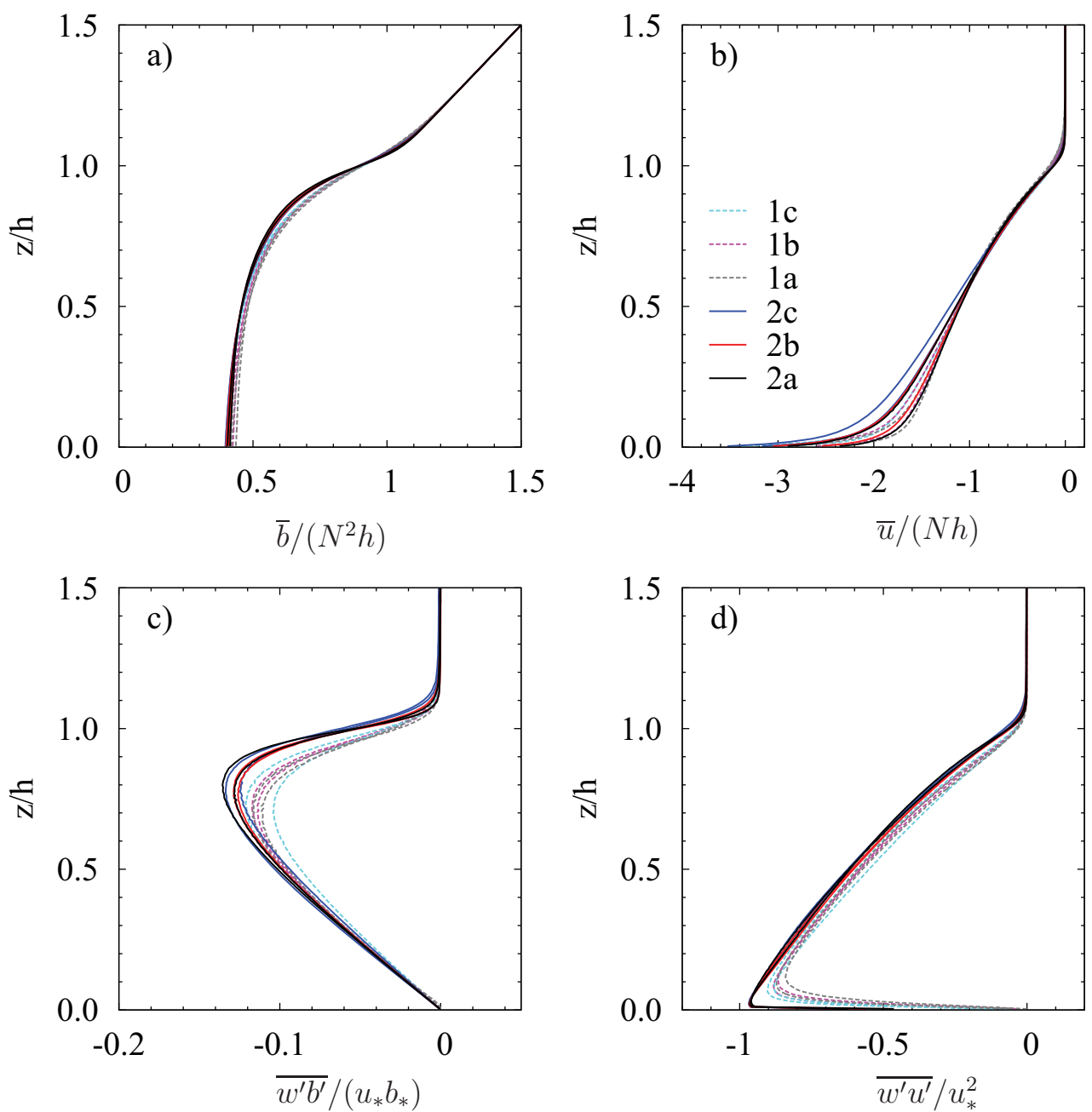

FIGURE 5. Self-similar profiles after rescaling. a) mean buoyancy; b) mean horizontal velocity; c) buoyancy flux; d) momentum flux. Profiles have been averaged for $N t>40$ until the end of the simulation in two parts, yielding two average profiles per simulation. Solid lines: high Reynolds number cases (2a-2c), see table 1; dashed lines: low Reynolds number cases (1a-1c).

\section{Self-similarity implies $h \propto \sqrt{t}$}

Next, we show that the observation of self-similarity is sufficient to establish the entrainment rate. We average $(2.1,2.2)$ over the homogeneous directions and obtain:

$$
\begin{aligned}
& \frac{\partial \bar{u}}{\partial t}+\frac{\partial \overline{w^{\prime} u^{\prime}}}{\partial z}=\nu \frac{\partial^{2} \bar{u}}{\partial z^{2}} \\
& \frac{\partial \bar{b}}{\partial t}+\frac{\partial \overline{w^{\prime} b^{\prime}}}{\partial z}=\kappa \frac{\partial^{2} \bar{b}}{\partial z^{2}} .
\end{aligned}
$$

Integrating (4.1) over $z$, letting $R e_{b} \rightarrow \infty$ and using the boundary conditions results in

$$
\frac{\mathrm{d}}{\mathrm{d} t} \int_{0}^{\infty} \bar{u} \mathrm{~d} z=-u_{*}^{2},
$$



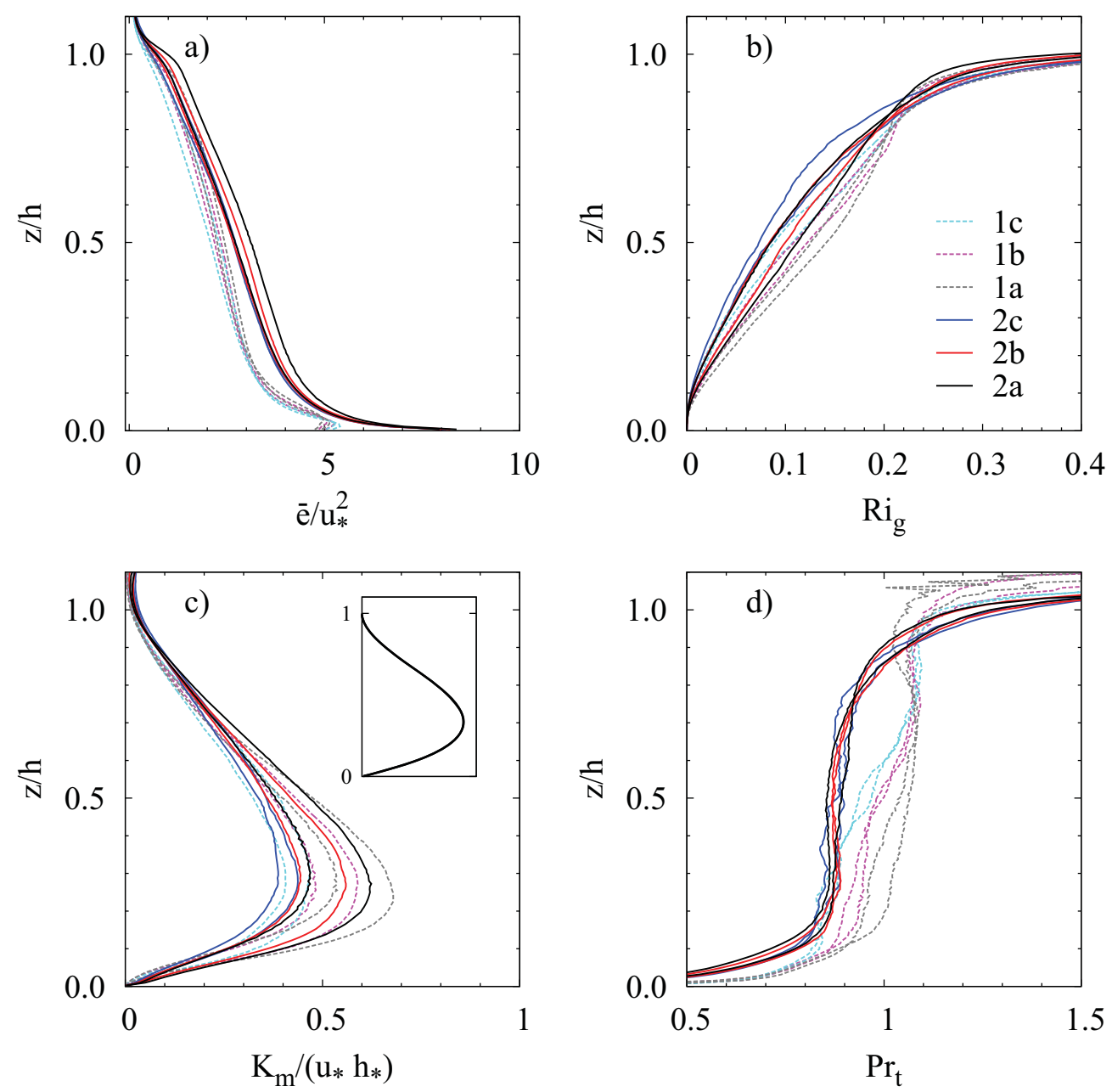

FiguRE 6. a) scaled TKE profile $\bar{e}=\left(\overline{u^{\prime 2}}+\overline{v^{\prime 2}}+\overline{w^{\prime 2}}\right) / 2$. b) gradient Richardson number; c) scaled eddy viscosity profile; inset: shape function of eddy viscosity employed in the KPP method. (d) turbulent Prandtl number $\mathrm{Pr}_{t}$. Profiles have been averaged for $N t>40$ until the end of the simulation in two parts, yielding two average profiles per simulation. Solid lines: high Reynolds number cases (2a-2c), dashed lines: low Reynolds number cases (1a-1c). For details, see table 1.

By substituting the self-similarity ansatz

$$
\begin{array}{rlrl}
\bar{u}(z, t) & =u_{*}(N t)^{\alpha_{u}} f_{u}(\eta), & \bar{b}(z, t) & =b_{*}(N t)^{\alpha_{b}} f_{b}(\eta), \\
\overline{w^{\prime} u^{\prime}}(z, t) & =u_{*}^{2}(N t)^{\alpha_{w u}} f_{w u}(\eta), \overline{w^{\prime} b^{\prime}}(z, t) & =u_{*} b_{*}(N t)^{\alpha_{w b}} f_{w b}(\eta), \\
\eta & =\frac{z}{h(t)}, & h(t) & =h_{*}(N t)^{\alpha},
\end{array}
$$

into (4.1-4.3) one obtains

$$
\alpha_{u}=1-\alpha, \quad \alpha_{w b}=\alpha_{b}+\alpha-1, \quad \alpha_{w u}=\alpha_{u}+\alpha-1=0 .
$$

In addition, since $b(z, t)$ is always linear for $z \gg h(t),(4.4)$ implies that $\alpha_{b}=\alpha$.

The self-similarity analysis therefore immediately pins down the observed scaling exponents for $\overline{w^{\prime} u^{\prime}}$ and provides a direct relation between the scaling of $h, \bar{u}, \bar{b}$ and $\overline{w^{\prime} b^{\prime}}$, 
respectively, but the exponent $\alpha$ - the primary unknown - remains elusive. To obtain additional information about $\alpha$ we turn to the budget of turbulence kinetic energy, given by

$$
\frac{\partial \bar{e}}{\partial t}=-\frac{\partial \bar{T}}{\partial z}-\overline{w^{\prime} u^{\prime}} \frac{\partial \bar{u}}{\partial z}+\overline{w^{\prime} b^{\prime}}-\varepsilon
$$

where $-\partial \bar{T} / \partial z$ is the transport of TKE (including transport by pressure correlations) and $\varepsilon$ is the dissipation rate. As before we assume self-similar profiles of the form

$$
\bar{e}(z, t)=u_{*}^{2}(N t)^{\alpha_{e}} f_{e}(\eta), \quad \bar{T}(z, t)=u_{*}^{3}(N t)^{\alpha_{T}} f_{T}(\eta), \quad \varepsilon(z, t)=N u_{*}^{2}(N t)^{\alpha_{\varepsilon}} f_{\varepsilon}(\eta) .
$$

Substitution into (4.6) and division by $N u_{*}^{2}$ yields

$$
(N t)^{\alpha_{e}-1}\left[\alpha_{e} f_{e}-\alpha \eta f_{e}^{\prime}\right]=-(N t)^{\alpha_{T}-\alpha} f_{T}^{\prime}-(N t)^{1-2 \alpha} f_{w u} f_{u}^{\prime}+(N t)^{2 \alpha-1} f_{w b}-(N t)^{\alpha_{\varepsilon}} f_{\varepsilon},
$$

where a prime here means differentiation to $\eta$ : $f^{\prime}=\mathrm{d} f / \mathrm{d} \eta$. The entrainment process entails a conversion from kinetic to potential energy which is represented in (4.8) by $(N t)^{2 \alpha-1} f_{w b}$. This negative term plays a central role in the TKE budget and must be balanced by part of the shear production term, which scales as $(N t)^{1-2 \alpha}$. The possibility that $\alpha>1 / 2$ can be ruled out, because production by shear would vanish while destruction by buoyancy increases, which cannot be sustained. On the other hand, if $\alpha<1 / 2$, the buoyancy flux vanishes while the shear production grows in time. The dissipation rate would have to keep up to balance the increasing shear production; but the scenario of a vanishing buoyancy flux is unlikely because the shear production would significantly enhance entrainment, thus increasing the magnitude of the buoyancy flux. One can therefore conclude that

$$
\alpha=\frac{1}{2} .
$$

The analysis shows that the observed self-similar behaviour can only be consistent with a mixed layer growing according to $h \sim t^{\frac{1}{2}}$. Kundu (1981) performed a comparable self-similarity analysis of the problem and reached the same conclusion, even though he employed the assumption of a constant gradient Richardson number at $z=h$ (Pollard et al. 1973) to close the system (see also §5). It is interesting that both approaches (tke-budget and constant gradient Richardson number) lead to identical scaling results; perhaps the tke-budget approach outlined above should be viewed as a constant fluxRichardson number closure.

Next we study the other terms in the budget. First, it seems reasonable to assume that the excess shear production will be balanced by dissipation, from which one can infer: $\alpha_{\varepsilon}=0$. This is an important conclusion, because one would model $\varepsilon$ typically by

$$
\varepsilon=\frac{u_{*}^{3}}{\ell_{\varepsilon}} .
$$

The statement $\alpha_{\varepsilon}=0$ implies that the logical choice $\ell_{\varepsilon} \sim h$ would be inconsistent, since $\ell_{\varepsilon}$ must stay constant as both $\varepsilon$ and $u_{*}$ are constant. This leaves $\ell_{\varepsilon} \sim h_{*}$.

The remaining terms, viz. $t^{\alpha_{e}-1}$ and $t^{\alpha_{T}-\alpha}$ can either possess the same scaling properties, or be of lower order, such that they vanish (in relative sense) as time proceeds. The latter situation seems to be case for the first term of (4.8); indeed, looking at the behaviour of TKE in figure $6 \mathrm{a}$, we can conclude that $\alpha_{e}=0$, which means that the first term vanishes with time as $t^{-1}$. In the same vein one might expect that $\bar{T} \simeq \overline{w^{\prime} e^{\prime}} \sim u_{*}^{3}$ and therefore $\alpha_{T}=0$, which entails that the transport term $\partial T / \partial z$ decreases as $t^{-\frac{1}{2}}$. This has an interesting consequence since it suggests that as time proceeds the budget 


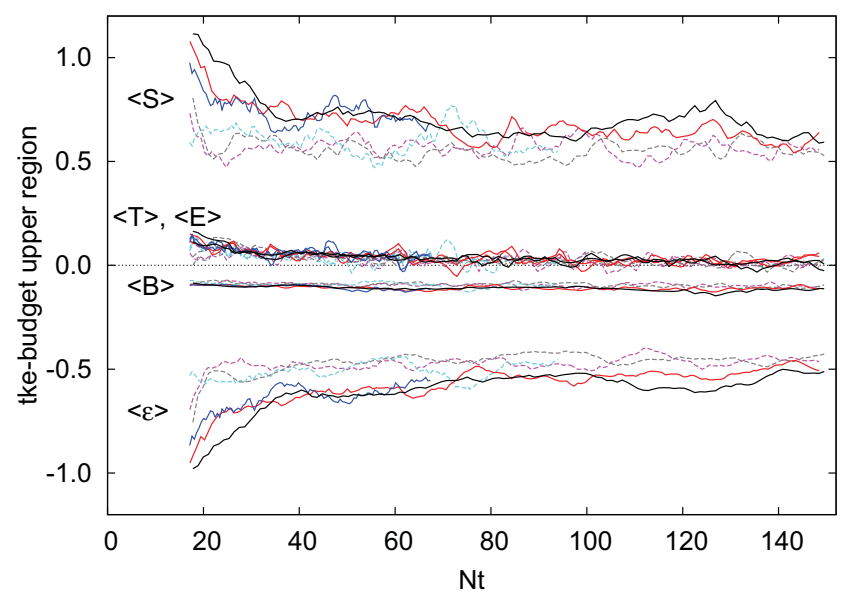

FiguRE 7. Budget of turbulence kinetic energy averaged over the upper half of the mixed layer $(h / 2<z<h)$. Solid lines: high Reynolds number cases (2a-2c), dashed lines: low Reynolds number cases (1a-1c). For details, see table 1.

becomes progressively more 'local' (Nieuwstadt 1984), i.e. that the balance will be set by local production of TKE by shear and local destruction by buoyancy and dissipation.

To test the above inferences derived from self-similarity, we diagnose all terms of the TKE budget (4.6) in the DNS. To present the evolution of the various terms in time we average each term over the upper half of the mixed layer. By focussing on the upper part we avoid the influence of the viscous wall region near the bottom surface with different scaling behaviour which obfuscates the self-similar behaviour of the entrainment zone. Indeed, in figure $5 \mathrm{~b}$ one can observe that the lower half of the $\bar{u}$-profile is still in a transient phase because the viscous layer does not scale with $h(t)$, whereas for $z / h>0.5$ the scaled profiles are stationary and self-similar. After averaging we additionally nondimensionalize each term by $u_{*}^{3} / h_{*}$. For example for the dissipation term we calculate

$$
\langle\varepsilon\rangle=\frac{1}{N u_{*}^{2}} \frac{2}{h} \int_{h / 2}^{\infty} \varepsilon(z, t) d z .
$$

In the same way we diagnose $\langle E\rangle$ based on $\partial \bar{e} / \partial t,\langle T\rangle$ based on the transport term, $\langle S\rangle$ on the shear production term, and $\langle B\rangle$ on the buoyancy term. The evolution of the budget terms in figure 7 reveals that the upper layer has converged at about $N t \simeq 40$ but also that the Reynolds number effects are very modest. The graph also shows that the balance is primarily constituted by shear, dissipation, and buoyancy, whereas the transport terms are very small, implying that the former terms are in balance locally. These results are clearly in agreement with the results from the self-similarity analysis. In figure 8 we present the full profiles in the upper layer. These profiles were first rescaled and subsequently averaged over time (for $N t>40$ ). In addition we have lumped (averaged) the high Reynolds number simulations and the low Reynolds number simulations. The graphs look qualitatively quite the same and convey the message that shear production is in local balance with dissipation and destruction by buoyancy (Nieuwstadt 1984).

\section{Discussion}

It has become clear that the DNS data do not support the entrainment law $E \propto R i^{-1}$ as found by KP69. In their paper, however, the authors provide an energy flux argument why 

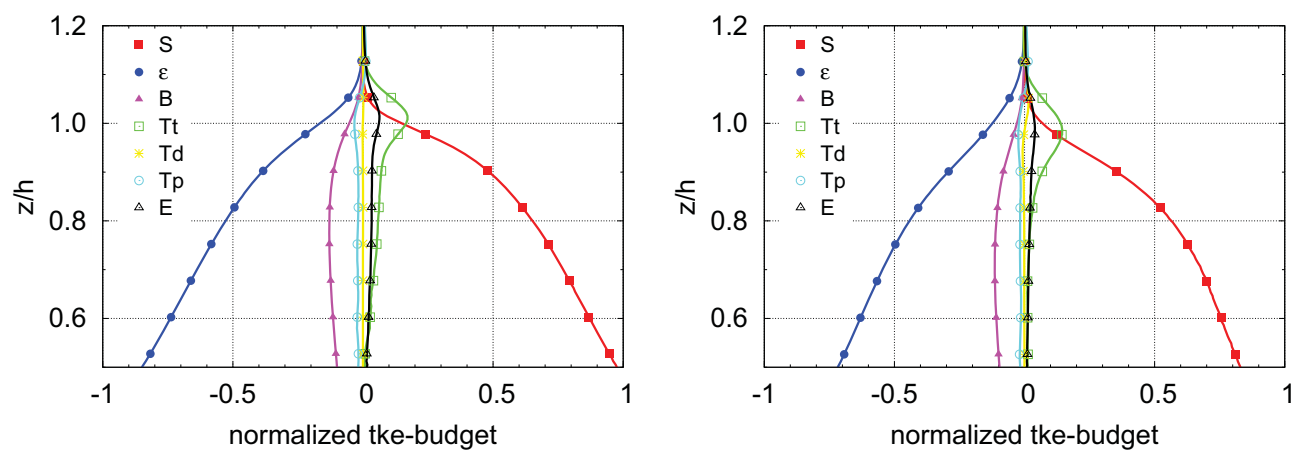

Figure 8. Profiles of the tke-budget normalized by $u_{*}^{3} / h_{*}$. Left: average of the three high Reynolds number cases. Right: average of the three low Reynolds number cases. S: shear production, $\varepsilon$ dissipation rate, B: buoyancy production, Tt: turbulent transport, Td: viscous transport, Tp: pressure transport, E: storage.

one should expect a -1-law for this experiment. Probably the caveat in their reasoning is related to the dominant length scale of the flow, which they assumed to be $h$. To highlight the important role of the length scale, below we present an alternative derivation for the entrainment law. First assume a balance between production of turbulence kinetic energy by shear and destruction by dissipation and buoyancy:

$$
-\overline{w^{\prime} u^{\prime}} \frac{\partial \bar{u}}{\partial z} \simeq-\overline{w^{\prime} b^{\prime}}+\varepsilon .
$$

Invoking a zero-order jump model $\overline{w^{\prime} b^{\prime}}=-w_{e} \Delta b=-w_{e} N^{2} h / 2$ and scaling both the shear production term and the dissipation rate as $u_{*}^{3} / \ell$ gives

$$
E=\frac{w_{e}}{u_{*}} \sim \frac{2 h_{*}^{2}}{h \ell} .
$$

KP69 opted for the natural choice $\ell \sim h$, which then together with (3.1) immediately gives $E \sim R i^{-1}$. However, opting for the other natural choice for the length scale, $\ell \sim h_{*}$, directly gives $E \sim R i^{-\frac{1}{2}}$. Apparently, the turbulence in this flow is characterised by the confined length scale which does not increase with the depth of the mixed layer. This finding is consistent with our earlier conclusion with respect to the dissipation length scale $(\S 4)$ where we found that $l_{\varepsilon} \sim h_{*}$.

As mentioned in $\S 1$, several authors, including e.g. Pollard et al. (1973) and Price (1979), advocated that for a better understanding of the flow one should also consider a Richardson number based on the mean mixed layer flow $V$ rather than $u_{*}$. Pollard et al. (1973) used $R i_{v}$ to close their mixed layer equations which leads to the $R i^{-\frac{1}{2}}$ law; their argument is based on the idea that the flow will strive to make $R i_{v}$ constant. If $R i_{v}$ drops below this value, then turbulence will be enhanced leading to higher values of $h$ and $\Delta b$, thus restoring $R i_{v}$. A high value of $R i_{v}$ would reduce turbulence and therefore also $R i_{v}$. We can check this idea in the DNS data by diagnosing $R i_{v}$. There is some arbitrariness in defining $V$ because the $\bar{u}$ profile deviates substantially from an ideal well mixed profile and since the lower part will be dominated viscosity near the wall. Hence we chose to take an average over the upper half of the mixed layer $V=2 h^{-1} \int_{h / 2}^{h} \bar{u} d z$. The result for $R i_{v}$, plotted in figure 9, shows that $R i_{v}$ indeed becomes constant as hypothesized by Pollard et al. (1973). In addition it turns out to be close to the value of $R i_{v}=0.6$ which Price (1979) extracted from the laboratory experiments of KP69 and Kantha et al. 


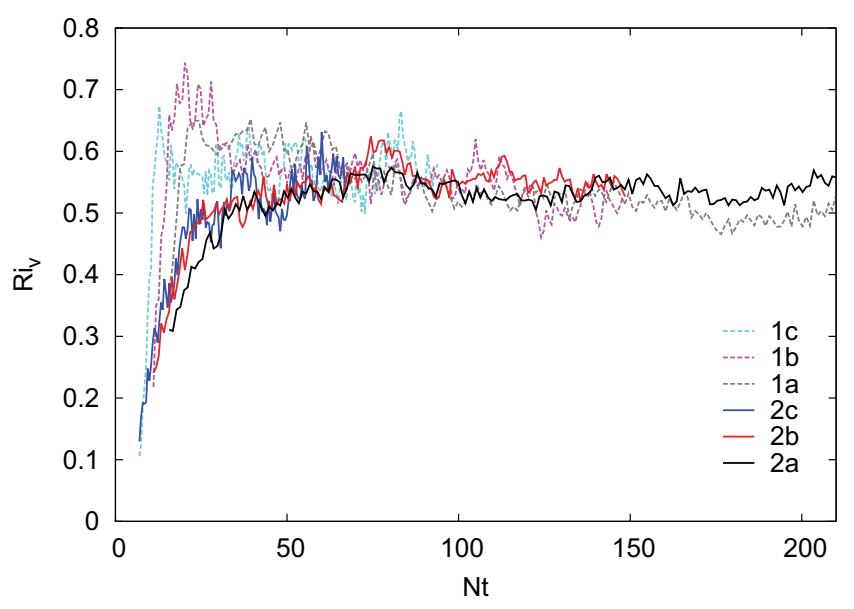

Figure 9. Evolution of $R i_{v}$ defined in (1.3). Solid lines: high Reynolds number cases (2a-2c), dashed lines: low Reynolds number cases (1a-1c). For details, see table 1.

(1977). For a linear initial stratification, Price (1979) derived that $E=\frac{1}{2} R i_{v}^{\frac{1}{2}} R i^{-\frac{1}{2}}$, i.e. $E \simeq 0.38 R i^{-\frac{1}{2}}$, which is close to our findings (3.4). Note that for the growth law we found, $h=h_{*} \sqrt{N t}$, a simple mixed layer perspective shows that $R i_{v}=1 / 2$ should be expected, since integration of $d(h V) / d t=u_{*}^{2}$, gives $V=u_{*}^{2} t / h$, and hence $R i_{v}=1 / 2$.

Pollard et al. (1973) also contended that the entrainment in this flow is not caused by turbulence created at the surface which propagates to the density interface to induce entrainment; rather it is the existence of a mean velocity difference across the interface that produces local turbulence which drives entrainment. This view is substantiated by the DNS data of the tke-budget (Figs 7 and 8) which reveal that the transport terms are small.

\section{Conclusions}

Using Direct Numerical Simulation we have studied the deepening of a shear-driven turbulent layer penetrating into a linearly stratified quiescent layer. The simulation design was inspired by the classical laboratory experiments of Kato \& Phillips (1969) by prescribing a constant wall shear stress and using an initial linear stratification, but the design also avoids unwanted side wall and rotation effects. Upon proper scaling, the mixed layer depths in the different simulations are found to universally deepen as a function of the square root of time, independent of the initial stratification and the Reynolds number of the simulations, provided that the Reynolds number is large enough. The dimensionless entrainment velocity is found to vary with the bulk Richardson number as $E \sim R i^{-\frac{1}{2}}$. These results differ from the conclusions of Kato \& Phillips $(1969)\left(E \sim R i^{-1}\right)$, but agree with the views of later studies by e.g. Pollard et al. (1973) and Price (1979). In line with these and other studies such as Thompson (1979); Deardorff \& Willis (1982); Scranton \& Lindberg (1983); Jones \& Mulhearn (1983); Deardorff \& Yoon (1984), it can be concluded that the entrainment results in rotating annulus set-ups suffered from a number of inherent but unwanted side effects, such as friction at side walls, tilting of the water level, and generation of secondary flows due to rotation. The DNS enabled us to study the original case in a purer form and revealed that the rescaled profiles of first and higher order moments attain universal (self-similar) shapes. A self-similarity analysis of the conservation equations and the tke-budget showed that only a square root growth law 
is consistent with self-similar behaviour. This result agrees with the self-similarity analysis of Kundu (1981) which was based on the closure of a constant gradient Richardson number (Pollard et al. 1973).

Of course the buoyancy Reynolds numbers atmospheric/oceanic situations are much higher than studied here. However the study shows that the results converge to self-similar profiles when the Reynolds number becomes sufficiently high. As these self-similar profiles are Reynolds-number independent, the implication is that these profiles are representative for atmospheric/oceanic situations, that is, for the ideal situations of spatial homogeneity and in absence of other forcings such as the Coriolis force and surface buoyancy fluxes. In order to be a closer analogy to the oceanic/atmospheric mixed layer, these effects should be considered (e.g. Conzemius \& Fedorovich (2006); Beare et al. (2006)). In addition, it is interesting to replace the fixed surface stress boundary condition by a constant no-slip velocity and, because $u_{*}$ is no longer a constant, study the impact on the entrainment law (3.4).

Finally we note that a shear-less convective boundary layer, driven by a constant surface buoyancy flux and entraining into a linearly stratified environment, also displays a square root growth law (Tennekes 1973)

$$
h(t)=\sqrt{2(1+2 A) \frac{B_{0} t}{N^{2}}}
$$

where $A \simeq 0.25$ is the entrainment flux ratio and $B_{0}$ the surface buoyancy flux. This is remarkable to the extent that the entrainment rate has a different dependence on the Richardson number $E=A R i^{-1}$. Equation (6.1) allows one to make a direct quantitative comparison between the effectivity of growth by a surface buoyancy flux and the growth by a surface momentum flux as given by (3.4). It follows that the layers grow at the same rate when $B_{0}=u_{*}^{2} N / 3$. For $u_{*}=0.1 \mathrm{~ms}^{-1}$ and $N$ based on a typical temperature lapse rate of $5 \cdot 10^{-3} \mathrm{Km}^{-1}$, one finds $B_{0}=4 \cdot 10^{-5} \mathrm{~m}^{2} \mathrm{~s}^{-3}$, which corresponds to a surface heat flux on the order of $1 \mathrm{Wm}^{-2}$. Conversely, a heat flux of $130 \mathrm{Wm}^{-2}$ would correspond to $u_{*}=1 \mathrm{~ms}^{-1}$.

\section{Acknowledgements}

The authors gratefully acknowledge the following supercomputing grants: a PRACE (Partnership for Advanced Computing in Europe) grant for computations on the BlueGene in Juelich (Germany); an EPSRC (UK) grant for computations on HECToR; an NWO/NCF (Netherlands) grant for computations on Huygens. The National Center for Atmospheric Research is sponsored by the National Science Foundation. Dr B. van de Wiel is thanked for contributing interesting insights on the subject.

\section{REFERENCES}

Beare, Robert J, Macvean, Malcolm K, Holtslag, Albert Am, Cuxart, Joan, Esau, Igor, Golaz, Jean-Christophe, Jimenez, Maria A, Khairoutdinov, Marat, KosoVic, Branko, Lewellen, David et al. 2006 An intercomparison of large-eddy simulations of the stable boundary layer. Boundary-Layer Meteorology 118 (2), 247-272.

Conzemius, Robert J. \& Fedorovich, Evgeni 2006 Dynamics of sheared convective boundary layer entrainment. part II: evaluation of bulk model predictions of entrainment flux. $J$. Atmos. Sci. 63, 1179-1199.

Deardorff, J. W. \& Willis, G. E. 1982 Dependence of mixed-layer entrainment on shear stress and velocity jump. J . Fluid Mech. 115, 123-149. 
Deardorff, J. W. \& Yoon, S.-C. 1984 On the use of an annulus to study mixed-layer entrainment. J. Fluid Mech. 142, 97-120.

Fernando, H. J. S. 1991 Turbulent mixing in stratified fluids. Annu. Rev. Fluid Mech. 23, $455-493$.

Galperin, B., Kantha, L. H., Hassid, S. \& Rosati, A. 1988 A quasi-equilibrium turbulent energy model for geophysical flows. J. Atmos. Sci. 45, 55-62.

Jones, I. S. F. \& MulheARn, P. J. 1983 The influence of external turbulence on sheared interfaces. Geophys. Astrophys. Fluids Dynamics 24, 49-62.

Kantha, L. H., Phillips, O. M. \& AzAd, R. S. 1977 On turbulent entrainment at a stable density interface. J. Fluid Mech. 79, 753-768.

Kato, H. \& Phillips, O. M. 1969 On the penetration of a turbulent layer into stratified fluid. Journal of Fluid Mechanics 37 (04), 643-655.

Kundu, P. K. K. 1981 Self-similarity in stress-driven entrainment experiments. Journal of Geophysical Research 86 (C3), 1979-1988.

Large, W. G., McWilliams, J. C. \& Doney, S. C. 1994 Oceanic vertical mixing: A review and a model with a nonlocal boundary layer parameterization. Reviews of Geophysics 32 (4), 363-403.

Ligniéres, F., F., Califano \& A., Mangeney 1998 Stress-driven mixed layer in a stably stratified fluid. Geophys. Astrophys. Fluid Dynamics 88, 81-113.

Mellor, G. L. \& Durbin, P. A. 1975 The structure and dynamics of the ocean surface mixed layer. J. Phys. Oceanogr. 5, 718-728.

Nieuwstadt, F. T. M. 1984 The turbulent structure of the stable, nocturnal boundary layer. Journal of the Atmospheric Sciences 41 (14), 2202-2216.

Pollard, R. T., Rhines, P. B. \& Thompson, R. O. R. Y. 1973 The deepening of the windmixed layer. Geophys. Fluid. Dyn. 3, 381-404.

PRICE, J. F. 1979 On the scaling of stress-driven entrainment experiments. J. Fluid Mech. 90, $509-529$

Price, J. F. 1981 Upper ocean response to a hurricane. J. Phys. Oceanogr. 11 (4), 153-175.

VAN REEUWIJK, M. 2007 Direct simulation and regularization modeling of turbulent thermal convection. PhD thesis, Delft University of Technology.

van Reeuwijk, M., Jonker, H. J. J. \& Hanjalić, K. 2008 Wind and boundary layers in Rayleigh-Bénard convection. i. analysis and modelling. Phys. Rev. E 77, 036311.

Scranton, D. R. \& Lindberg, W. R. 1983 An experimental study of entraining, stressdriven, stratified flow in an annulus. Phys. Fluids 26, 1198-1205.

Stull 1998 An introduction to boundary layer meteorology. Kluwer Academic Publishers.

Sullivan, P. P., Moeng, C.-H., Stevens, B., Lenschow, D. H. \& Major, S. D. 1998 Structure of the entrainment zone capping the convective atmospheric boundary layer. $J$. Atmos. Sci. 55, 3042-3064.

Tennekes, H. 1973 A model for the dynamics of the inversion above a convective boundary layer. Journal of the Atmospheric Sciences 30 (4), 558-566.

Thompson, R. O. R. Y. 1979 A re-examination of the entrainment process in some laboratory flows. Dyn. Atmos. Oceans 4, 45-55.

Thorpe, S. A. 2005 The turbulent ocean. Cambridge University Press.

Troen, I. \& MAhrt, L. 1986 A simple model of the atmospheric boundary layer; sensitivity to surface evaporation. Bound.-Layer Meteorol. 37, 129-148.

Verstappen, R. W. C. P. \& Veldman, A. E. P. 2003 Symmetry-preserving discretization of turbulent flow. J. Comput. Phys. 187 (1), 343-368. 